\title{
Monitoring of Tissue Ablation Using Time Series of Ultrasound RF Data
}

\author{
Farhad Imani ${ }^{1}$, Mark Z. Wu ${ }^{1, \star}$, Andras Lasso ${ }^{1}$, Everett C. Burdette ${ }^{3}$, \\ Mohammad Daoud ${ }^{2}$, Gabor Fitchinger ${ }^{1}$, \\ Purang Abolmaesumi ${ }^{2}$, and Parvin Mousavi ${ }^{1}$ \\ 1 Queen's University, Canada \\ 2 University of British Columbia, Canada \\ 3 Acoustic MedSystems Incorporation, USA
}

\begin{abstract}
Purpose: This paper is the first report on the monitoring of tissue ablation using ultrasound RF echo time series. Methods: We calcuate frequency and time domain features of time series of RF echoes from stationary tissue and transducer, and correlate them with ablated and non-ablated tissue properties. Results: We combine these features in a nonlinear classification framework and demonstrate up to $99 \%$ classification accuracy in distinguishing ablated and non-ablated regions of tissue, in areas as small as $12 \mathrm{~mm}^{2}$ in size. We also demonstrate significant improvement of ablated tissue classification using RF time series compared to the conventional approach of using single RF scan lines. Conclusions: The results of this study suggest $\mathrm{RF}$ echo time series as a promising approach for monitoring ablation, and capturing the changes in the tissue microstructure as a result of heat-induced necrosis.
\end{abstract}

Keywords: tissue ablation, ablation monitoring, RF timer series.

\section{Introduction}

Primary and metastatic cancers of the liver cause significant distress, drastically decrease life expectancy for patients worldwide, and have an increasing frequency in North America [1. Surgical resection, and in some cases liver transplantation, are amongst potential interventions; however, only a small proportion of patients are candidates for these treatments [2. As a result, there has been increasing interest in ablation, including chemical ablation, cryoablation, and heat ablation, as an alternative mode of therapy 11. Ablation therapy can be focused to specific tumor locations, thus preserving most of the surrounding tissue. In addition to metastatic disease, multiple primary tumors may not be resectable. As a result, ablation can be utilized as a minimally invasive procedure, increasing the number of patients that can receive therapy.

In heat ablation therapy, the ablation applicator is placed within the target tissue and heat is transferred to the surrounding tissue causing necrosis of the

\footnotetext{
* Farhad Imani and Mark Z. Wu contributed equally to this work.
} 
target area. Microwave, laser, and Radio Frequency (RF) signals [1] have all been used as sources for heat ablation, with RF ablation being the method of choice for many clinicians. Despite advances in ablation therapy, real-time monitoring of the extent of ablation is a major limitation. The need to monitor the ablation zone is the key step to avoiding necrosis of healthy tissue and complete targeting of primary or metastatic tumors. Non-invasive monitoring techniques using magnetic resonance imaging (MRI), computed tomography (CT) and ultrasound imaging have been used for this purpose. A series of T1 and T2 MRI measurements were found to characterize changes in a variety of ex vivo tissues as a result of thermal coagulation [3], while computed tomography (CT) has been shown to effectively monitor RF ablation zones in porcine liver [10. Despite these successes, MRI and CT-based ablation monitoring suffer several drawbacks, including requiring specialized equipment for compatibility, limitations for real-time application, and universal availability.

Ultrasound-based ablation monitoring has garnered interest due to its wide availability and relative low cost. Conventional B-mode imaging does not clearly indicate the ablation zone, and in some cases can overestimate the extent of ablation [9]. Ultrasound elastography has emerged as a technique to augment conventional images for monitoring the ablation zone [9]. Following a mechanical stimulus, various elastic properties of the tissue have been calculated from ultrasound images to identify stiffer ablated lesions.

Another mechanism to augment conventional ultrasound is to utilize raw RF signals; these signals contain tissue typing information and have been previously used for monitoring changes in frequency-dependent attenuation to determine temperature 4. RF time series signals have also been effectively used for tissue typing at both high- and clinical-frequencies [8]. RF time series was acquired from stationary tissue and transducer over a few seconds. Frequency domain features and fractal dimension of these signals were used to distinguish between various tissue types, including healthy and cancerous prostate tissue [8].

In this paper, we aim to classify ablated and non-ablated regions of interest (ROI) in animal tissue following thermal ablation using time and frequency domain features of calibrated RF time series data. We show that the classification accuracies obtained by these features significantly outperform those achieved by spectral analysis of individual RF scan lines. The remainder of this paper is organized as follows: Section 2 presents the ablation data collection setup, feature extraction, and tissue classification approaches. Section 3 presents the results and discussion followed by concluding remarks and a summary in Section 4.

\section{Methods}

To differentiate between ablated and non-ablated tissue using RF time series, we acquired data from homogeneous, fresh chicken breast tissue [12. Three experiments were performed to collect data for monitoring the ablation process. An ultrasound intersitial thermal therapy (USITT) applicator (Acoustic MedSystems, Champaign, IL) is used for tissue coagulation. In each experiment, a piece 


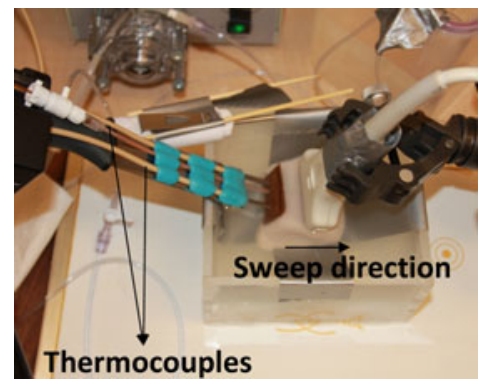

Fig. 1. Tissue ablation and data acquisition setup

of lean chicken breast tissue is placed in a warm water bath $\left(37^{\circ} \mathrm{C}\right)$ with generous water flow surrounding the tissue as depicted in Fig. 1. The water bath is then placed on a warming plate to ensure the temperature stays constant. The ablator is attached to two multi-sensor type T thermocouples (Physitemp Instruments, Clifton, NJ), and inserted in the tissue. The thermocouples are used to monitor the temperature of the tissue during ablation; each thermocouple contains three sensors located at the tip, and $0.5 \mathrm{~cm}$ and $1 \mathrm{~cm}$ from the tip. A water pump is used to cool the ablator with a water flow rate of $25 \mathrm{ml} / \mathrm{min}$. The ablator is configured to transmit RF waves with a power of $21.5 \mathrm{~W}$. Ablation is initiated while the thermal dose to the tissue increases. The experiment continues until at least one sensor displays a reading of $45^{\circ} \mathrm{C}$.

$\mathrm{RF}$ time series data is acquired at multiple points during the experiments: prior to ablation, at the end of ablation, and after a cooling period following ablation. Sample B-mode images prior to and at the end of ablation are shown in Figure 2. As seen, the ablation zone is not obvious in the B-mode images. Ultrasound data acquisition is performed using an Ultrasonix RP scanner (Ultrasonix, Richmond, BC) with a L14-5/38 linear array transducer (Ultrasonix, Richmod, $\mathrm{BC}$ ) operating at a central frequency of $6.6 \mathrm{MHz}$. The maximum depth of imaging is $4.5 \mathrm{~cm}$ while the focal point is set to $2 \mathrm{~cm}$. For RF time series acquisition, 178 frames of RF data are acquired at a frame rate of 45 frames/sec. Each $\mathrm{RF}$ frame has 256 scan lines in the lateral direction, each with 1080 samples in the axial direction. The coupling medium for ultrasound imaging is water.

For calibration purposes, $\mathrm{RF}$ data is also collected from a thick piece of glass placed in the water bath at the focal point of the transducer, with imaging parameters identical to the ablation experiments.

\subsection{ROI Selection and Feature Extraction}

To characterize ablated and non-ablated tissue, frequency and time domain features of calibrated RF time series are calculated over ROIs. RF time series from cooled tissue at the end of ablation is used for our analysis. After aligning the orientation of the ultrasound and histology images, the tip of the ablator is used as a landmark for selection of the ROIs. Tissue characterization is compared with that of spectral features of a single RF frame over the same ROIs. 


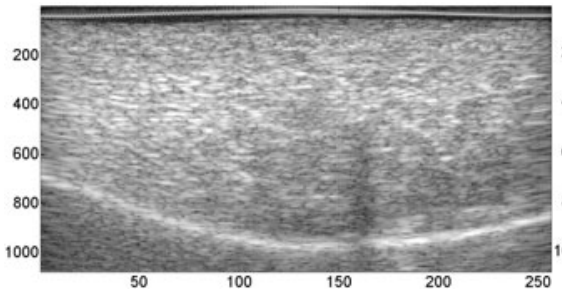

(a)

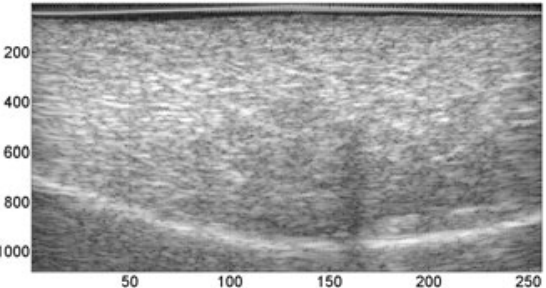

(b)

Fig. 2. B-mode images of the tissue (a) prior to, and (b) at the end of ablation. Note that the ablation zone is not visible on the ultrasound image after ablation.

A set of 120 equally-sized ROIs from ablated and non-ablated regions of the tissue are selected in each experiment, resulting in a total of 360 ROIs. The size of each ROI is 54 samples of RF from 4 scan lines that translate to a physical dimension of $5.93 \mathrm{~mm} \times 2.08 \mathrm{~mm}$. The ratio of the number of selected ablated to non-ablated ROIs is 1:1 (i.e. a total of 180 ablated and 180 non-ablated ROIs from three experiments). Following ablation, cooling, and data acquisition, the tissue specimen is cut approximately at the same plane as the ultrasound image acquisition, as seen in Fig. 3. The ablated zone of the tissue is clearly depicted. A crude manual registration approach is used to align ultrasound data with the image of the tissue slice at the same plane, and ROI selections are made from the ablated and non-ablated tissue accordingly.

$\mathrm{RF}$ time series data is calibrated for transducer effects, and used to extract features that are only related to the tissue properties. Calibration has been previously used in the literature prior to extracting spectral properties of RF ultrasound data to deconvolve the point spread function of the ultrasound imaging system [5]. Therefore, the deconvolved ultrasound RF signal is dominated by the response function of the tissue. Similarly, we use the RF data acquired from a piece of glass with high reflection. For each time frame of RF time series, its deconvolution with the glass RF signal is calculated and used as calibrated data. To compute this, the Fourier transform of a frame is divided by the Fourier transform of the glass RF signal; the calibrated RF data is computed by taking the inverse Fourier transform of this division. The time series data is then passed through a hamming window to remove the effect of artifacts in the frequency domain due to sharp edges of the rectangular windowing.

The calculated features of the calibrated RF time series are: i) the integral of the power spectrum of the first to the fourth quarter of its frequency range (Features 1-4); ii) the intercept and slope of a line fitted to the average power spectrum of the calibrated RF time series in each ROI (Features 5-6, respectively); iii) the trend of the power spectrum in different frequency bands captured by the slope of the power spectrum over the first to the fourth quarter of the frequency range (Features 7-10); and iv) the fractal dimension of the calibrated RF time series calculated using Higuchi's algorithm (Feature 11) [8]. 
Spectral analysis of single RF frames has been applied to tissue typing during the past decades [5. We evaluate the performance of RF time series for characterizing ablated tissue by comparing it to the performance of the spectral features of a single RF frame from cooled tissue at the end of ablation. To extract spectral features, each scan line of an RF frame is passed through a Hamming window, followed by calibration where the power spectrum of an ROI is divided by the power spectrum of the RF data from the glass. The intercept and the slope of the regression line to the power spectrum, and the average value of the spectrum over the measurement bandwidth (midband fit) constitute the spectral features (Features 12-14). It is worth mentioning that we compensate for the effect of depth on the slope and the midband fit features using a linear attenuation coefficient similar to [5].

\subsection{Classification}

A support vector machine (SVM) classifier is used to classify ablated and nonablated ROIs [6]. This classifier finds an optimal hyperplane with maximum margin separating the data into two classes. The parameters of this hyperplane are tuned by trying different kernel functions to map the training vector to higher dimensions. Among different kernel functions, the radial basis function is easier to initialize and outperforms others in terms of accuracy.

We follow a five-fold cross validation strategy. In other words, we randomly partition the ROIs into an $80 \%-20 \%$ ratio. We select $80 \%$ of the ROIs as training data and the remainder for testing, and repeat this for all 5 portions of the data. The entire process of five-fold cross validation is repeated 100 times, each time with random partitioning of the ROIs into five folds, to remove the effect of bias in data division. The mean classification accuracies and standard deviations are calculated over these 500 trials.

We also perform an exhaustive search for all 11 time series and three spectral features to find the optimal feature group in each category for characterizing the ablated tissue. To choose the optimal subset of 11 features, different subsets should be examined. We try subsets of 2-10 features of the time series automatically. The optimal search is done similarly for subsets of size two and three for the spectral features. The optimal classification accuracy and the best feature subset are found for each classification.

\section{Results and Discussion}

Ablated Tissue Classification: Two categories of features including RF time series and conventional single RF frame features are extracted from the ROIs. We performed a separate exhaustive search on each category and each experiment to identify optimal features for characterizing ablated tissue. In addition, we pooled all ROIs from the three experiments and searched for optimal features of RF time series and single RF frame.

Mean classification accuracies and standard deviations from 500 cross validation runs for individual experiments and pooled data are presented in Table 1. 


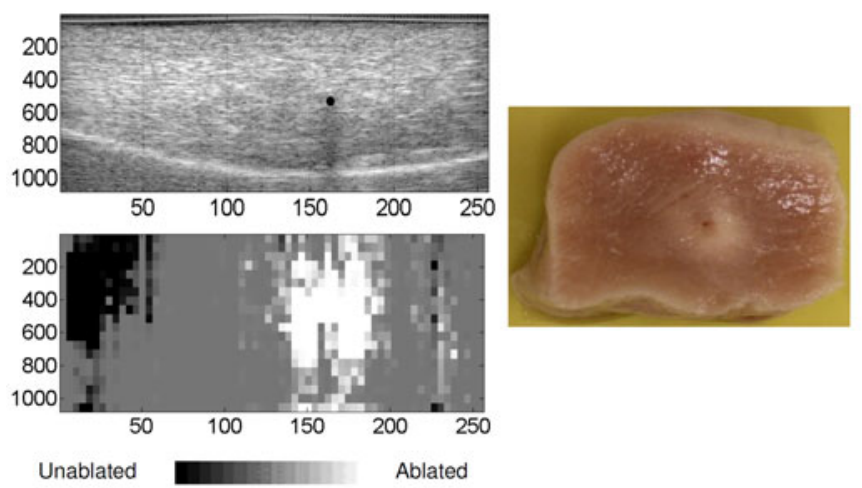

Fig. 3. An example of classifying entire image. Note the black circle in the B mode image indicating the location of the ablator, and the shadow underneath.

As seen in this table, almost perfect characterization of ablated and non-ablated tissue regions are made using only three to six RF time series features. The integral of the power spectrum of RF time series in the fourth quarter of the frequency band (Feature 4) is the single dominant feature for ablated tissue characterization in all three experiments and the pooled ROIs (rows 1-4 of the table). In addition, the intercept and the slope of the line fitted to the entire power spectrum, and the slope of the line fitted to the first frequency quarter of the power spectrum of the RF time series are the main features contributing to tissue typing. We calculated the Receiver Operating Characteristic (ROC) of tissue classification for pooled data from all experiments using the optimal time series features. The area under ROC curve obtained from RF time series features is close to one. Note that we tested RF time series data both immediately after ablation, and following a cool down period at the end of ablation. The results reported here are from the $\mathrm{RF}$ time series of cooled tissue; however results from the data at the end of ablation were very similar to those above.

In addition to classifying the ROIs of ablated and non-ablated tissue, we used the trained SVM classifier to test the accuracy of tissue characterization in the entire image. The B-mode ultrasound image, a color-map of the predicted posterior probabilities of ablation, and the tissue image at approximately the ultrasound imaging plane showing the physical extent of ablation are presented in Fig. 3. The ablation zone color-map is created by using all 11 features from ROIs in that particular experiment to train a model, which is then tested on the entire imaging plane. As seen, we are able to capture the ablated zone, using only a limited number of ROIs for training. The gray zone in the middle of the figure is mostly due to the deconvolution process involved in the calibration phase which results in discontinuity of the features between the RF scan lines.

\section{Comparison with Tissue Characterization Using Conventional Single} RF Frame: Similar to RF time series features, the combination of features from a single RF frame that results in the best ablated tissue characterization 
Table 1. Mean and standard deviation of classification accuracies using optimal RF time series features, and conventional RF spectral features

\begin{tabular}{c|c|c}
\hline Experiment & $\begin{array}{c}\text { Combination of time series } \\
\text { features }(\text { mean } \pm \text { std) }\end{array}$ & $\begin{array}{c}\text { Combination of spectral } \\
\text { features }(\text { mean } \pm \text { std) }\end{array}$ \\
\hline \hline Experiment 1 & Features $3,4,6,7,10,11$ & Features 12,13,14 \\
& $(99.09 \pm 0.24 \%)$ & $(82.3 \pm 2.84 \%)$ \\
\hline Experiment 2 & Features 3,4,5 & Features 12,14 \\
& $(98.01 \pm 0.64 \%)$ & $(92.05 \pm 1.69 \%)$ \\
\hline Experiment 3 & Features $4,5,6,7$ & Features 12,13,14 \\
& $(98.2 \pm 0.29 \%)$ & $(72.41 \pm 2.89 \%)$ \\
\hline Pooled Experiments & Features 4,5,6,7,9 & Features 12,13,14 \\
& $(99.29 \pm 0.24 \%)$ & $(75.59 \pm 1.28 \%)$ \\
\hline
\end{tabular}

are presented in Table 1. For most of the experiments as well as data from pooled experiments, all three spectral features are required. Comparing the columns of Table 1 shows that classification accuracies achieved using optimal time series features are above $98 \%$ for all of the experiments, whereas accuracies obtained incorporating the best spectral features vary between $72 \%$ and $92 \%$. Furthermore, the standard deviations of the classification accuracies obtained by time series features are superior as well. When the ROIs of three experiments are mixed, the classification accuracy obtained using the combination of time series features is $99.29 \%$. For the same ROIs, the classification accuracy computed by using spectral features is $75.59 \%$. Finally, the area under the ROC curve for the $\mathrm{RF}$ time series features is close to 1 compared to 0.8 for spectral features.

Previously, it was shown that temperature rise produced by acoustic propagation may be the major contributor to the ability of time series analysis for tissue typing [7. In other words, the speed of acoustic waves in the tissue is dependent on the tissue temperature. Ultrasound frames from RF time series can be used to estimate phase delay, hence virtual displacement. In our preliminary results, we have also observed that there is an upward trend for the phase delay of the ablated ROIs. Furthermore, the amplitude and frequency components of phase delay are distinguishable between ablated and non-ablated ROIs.

\section{Conclusion}

Monitoring the ablation zone is essential in ablation therapy to avoid necrosis of healthy tissue, and to completely target tumors. RF time series has shown great promise for tissue typing at both high and clinical frequencies. In this paper, we report for the first time the application of frequency and time domain features of RF time series for characterizing ablated tissue. We calibrate the RF signals of each individual frame to remove the contribution of the ultrasound imaging system. We calculate eleven features from the time series of RF signals of 360 ROIs in three animal experiments. We use an SVM classifier to characterize tissue into ablated and non-ablated categories, within a five-fold cross validation 
framework. The results show classification accuracies as high as $99 \%$ are obtained using these features by analyzing ROIs with sizes of $12 \mathrm{~mm}^{2}$. In addition, time series features significantly outperform spectral features of a conventional RF frame in terms of the classification mean accuracy and standard deviation, and the area under ROC curve. Future work will focus on expanding the current approach to real-time monitoring of the ablation zone in the entire imaging plane. Towards this goal, we extend the classification framework to the entire plane and demonstrate color-maps of the ablation zone that mimic the tissue histology closely. More work needs to be done on more accurate registration of the histology to ultrasound images, in addition to further experiments for generalization of the ablation zone beyond ROIs.

\section{References}

1. Nakakura, E.K., Choti, M.A.: Management of hepatocellular carcinoma. Oncology (Williston Park) 14(7), 1085-1102 (2000)

2. Steele, G.J.: Colorectal Cancer Metastatic to the Liver Resection. In: Cameron, J.L. (ed.) Current surgical therapy, 5th edn., pp. 283-289. St. Louis (1995)

3. Graham, S.J., Stanisz, G.J., Kecojevic, A., Bronskill, M.J., Henkelman, R.M.: Analysis of Changes in MRI Properties of Tissues After Heat Treatment. Magn. Reson. Med. 42(6), 1061-1071 (1999)

4. Ueno, S., Hashimoto, M., Fukukita, H., Yano, T.: Ultrasound Thermometry in Hyperthermia. In: Proc. of the IEEE Ultrasonics Symp., pp. 1645-1652 (1990)

5. Lizzi, F., Greenebaum, M., Feleppa, E., Elbaum, M.: Theoretical Framework for Spectrum Analysis in Ultrasonic Tissue Characterization. J. Acoust. Soc. Am. 73(4), 1366-1373 (1983)

6. A Library for SVMs, http://www.csie.ntu.edu.tw/ cjlin/libsvm/

7. Daoud, M.I., Mousavi, P., Imani, F., Rohling, R., Abolmaesumi, P.: Computeraided Tissue Characterization Using Ultrasound-induced Thermal Effects: Analytical Formulation and In vitro Animal Study. In: SPIE, Orlando (2011)

8. Moradi, M., Abolmaesumi, P., Siemens, D.R., Sauerbrei, E.E., Boag, A., Mousavi, P.: Augmenting Detection of Prostate Cancer in Transrectal Ultrasound Images Using SVM and RF Time Series. IEEE Trans. Biomed. Eng. 56(9), 2214-2223 (2009)

9. Boctor, E., deOliveira, M., Choti, M., Ghanem, R., Taylor, R., Hager, G., Fichtinger, G.: US Monitoring of Tissue Ablation Via Deformation Model and Shape Priors. In: Larsen, R., Nielsen, M., Sporring, J. (eds.) MICCAI 2006. LNCS, vol. 4191, pp. 405-412. Springer, Heidelberg (2006)

10. Cha, C.H., Lee, F.T., Gurney, J.M.: CT versus sonography for monitoring radiofrequency ablation in a porcine liver. Am. J. Roentgenol. 175, 705-711 (2000)

11. Lau, W.Y., Leung, T.W.T., Yu, S.C.H., Ho, S.K.W.: Percutaneous Local Ablative Therapy for Hepatocellular Carcinoma: A Review and Look Into the Future. Ann. Surg. 237(2), 171-179 (2003)

12. Zheng, X., Vaezy, S.: An Acoustic Backscatter-based Method for Localization of Lesions Induced by High-intensity Focused Ultrasound. Ultrasound in Med. and Biol. 36(4), 610-622 (2010) 